\title{
Milrinona inhalada en hipertensión pulmonar: A propósito de un caso
}

\author{
Case Report: Inhaled milrinone in pulmonary hypertension
}

Michelle Escobar ${ }^{1}$, Fernando Aranda ${ }^{1}$

\begin{abstract}
Introduction: Pulmonary hypertension $(\mathrm{PH})$ is a disease that is characterized for an elevated pressure in the pulmonary artery and an increased pulmonary vascular resistance (PVR). Inhaled milrinone has demonstrated acting as a selective pulmonary vasodilator, being a useful tool for the treatment of patients with PH in the perioperative. Materials and Methods: We report a successful case of inhaled milrinone in $\mathrm{PH}$ in cardiovascular surgery. The patient signed the informed consent for this report. Discussion: Patients with PH has increased risk of perioperative complications (mortality as far as 37-90\%) The management with intravenous vasodilators is frequently limited because of secondary effects of vasodilation and hypotension affecting the myocardial perfusion pressure. Milrinone is an inodilator that acts as an inhibitor of the phosphodiesterase III. Wang et al., and posterior studies have demonstrated that administered by inhalation it acts as a selective pulmonary vasodilator and inotrope, with a minor systemic effect. Conclusion: Inhaled milrinone have demonstrated to be a useful drug to lower PH, PVR and to enhance inotropism without deleterious systemic effects. Wide availability, lower costs and ease of administration make you think as it could be an ideal tool for perioperative management in patients with $\mathrm{PH}$. There are still more studies to define it's potentials.
\end{abstract}

\section{RESUMEN}

Introducción: La hipertensión pulmonar (HTP) es una enfermedad caracterizada por la elevación de las presiones de arteria pulmonar (PAP) y un aumento de la resistencia vascular pulmonar (RVP). La milrinona inhalada ha demostrado actuar como un vasodilatador pulmonar selectivo siendo una herramienta útil en el manejo de los pacientes con HTP en el perioperatorio. Materiales y Métodos: Reportamos un caso exitoso de milrinona inhalada en HTP en ciru-

\section{Key words:}

Pulmonary hipertension, milrinone, vasodilator agents, cardiovascular surgery

\section{Palabras clave:}

Hipertensión pulmonar, milrinona,

agentes vasodilatadores, cirugía cardiovascular

Universidad de Valparaíso.

Fecha de ingreso: 14 de abril de 2019

Fecha de aceptación: 25 de mayo de 2019

\section{ORCID}

https://orcid.org/0000-0003-3478-5988 
gía cardiovascular. La paciente firmó el consentimiento informado para este reporte. Discusión: Pacientes con HTP tienen mayor riesgo de complicaciones perioperatorias (mortalidad hasta 37-90\%). Su manejo con vasodilatadores intravenosos es frecuentemente limitado por sus efectos secundarios de vasodilatación e hipotensión, perjudicando la presión de perfusión miocárdica. La milrinona es un inodilatador que actúa como inhibidor de la fosfodiesterasa III. Wang et al., y estudios posteriores, han demostrado que administrada por vía inhalatoria actúa como un vasodilatador pulmonar selectivo e inótropo, con menor efecto sistémico. Conclusión: La milrinona inhalada ha demostrado ser una herramienta útil para la disminución de la PAP, RVP y mejoría del inotropismo, sin efectos sistémicos deletéreos. Su amplia disponibilidad, menor costo y facilidad de administración, hacen pensar que podría ser una herramienta útil para el manejo perioperatorio de los pacientes con HTP. Hacen falta más trabajos para definir sus potencialidades.

\section{Introducción}

a hipertensión pulmonar (HTP) es una enfermedad caracterizada por la elevación de las presio-nes de pulmón (PAP) y un aumento de la resistencia vascular pulmonar (RVP) resultando, finalmente, en una falla cardiaca derecha[1],[2]. Su prevalencia ha ido en aumento gracias a los avances en materia diagnóstica y de tratamiento, permitiendo así una expectativa de vida más larga a quienes la padecen. A pesar de los avances recientes se ha visto que los pacientes con HTP preexistente están en mayor riesgo de complicaciones perioperatorias[2],[3].

El manejo clásico de la HTP durante el perioperatorio se basa en la administración de vasodilatadores intravenosos, sin embargo, su uso se ve frecuentemente limitado por los efectos sistémicos secundarios de vasodilatación e hipotensión, perjudicando la perfusión miocárdica[4].

Otras apuestas terapéuticas se han enfocado en el uso de vasodilatadores por vía inhalatoria, que actuarían predominantemente en la circulación pulmonar, reduciendo los efectos sistémicos deletéreos[4]. Se ha propuesto el uso de óxido nítrico, prostaglandinas y levosimendán, cuya implementación se ha visto limitada por su costo elevado, difícil implementación y efecto rebote. La milrinona inhalada ha demostrado actuar como un vasodilatador pulmonar selectivo siendo una herramienta útil en el manejo de los pacientes con HTP en el perioperatorio[1].

A continuación, presentamos un caso exitoso de uso de milrinona inhalada en HTP en cirugía cardiovascular y una revisión que pretende entregar luces respecto a la fisiopatología de la HTP y la evidencia actual respecto al uso de milrinona inhalada para su manejo perioperatorio.

\section{Materiales y Métodos}

Reportamos un caso exitoso con uso de milrinona inhalada en HTP en cirugía cardiovascular. La paciente firmó el consentimiento informado para este reporte.

\section{Caso Clínico}

Mujer de 62 años, con enfermedad coronaria severa de 3 vasos, revascularizada hace 3 meses mediante puentes mamarios a la ADA (LIMA) y a la ACX (RIMA) y un puente venoso a ADP. Cursando en el postoperatorio con ICC descompensada hasta el edema pulmonar en dos ocasiones.

El ecocardiograma transtorácico preoperatorio, destaca válvula mitral de velos muy engrosados, con apertura normal y disminución de coaptación de los velos, con insuficiencia mitral (IM) severa, función ventricular conservada. Cavidades derechas normales con PAP sistólica calculada de $50 \mathrm{mmHg}$.

La coronariografía confirmó solo permeabilidad residual de LIMA.

Ingresa para recambio valvular mitral (RVM), realizándose anestesia general con monitoreo invasivo y ecocardiografía transesofágica (ETE). En ETE destaca marcado deterioro de la función VI. En contexto de cardiopatía coronaria se inicia apoyo con levosimendán $0,1 \mathrm{ug} / \mathrm{kg} / \mathrm{min}$ y adrenalina $0,01 \mathrm{ug} / \mathrm{kg} / \mathrm{min} \mathrm{EV}$.

Tras esternotomía, la paciente comienza con elevación progresiva de las PAP, agravamiento IM, hipo- 


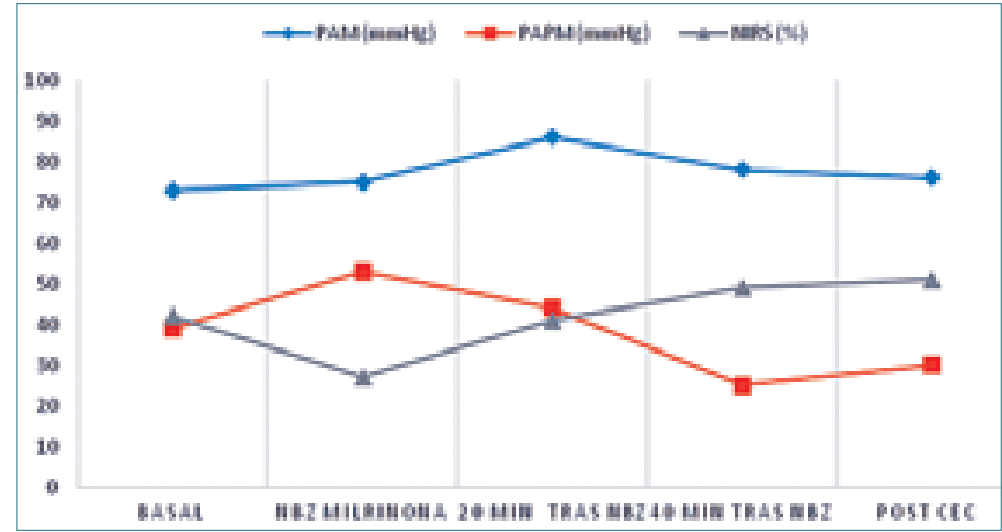

Figura 1. Evolución de los parámetros hemodinámicos; PAM: presión arterial media; PAPM: presión arterial pulmonar media; NIRS: espectroscopia por infrarrojo cercano; NBZ: nebulización con milrinona $0,025 \%$. tensión y falla del VD al ETE, sospechándose lesión de LIMA.

Debido a arteriopatía periférica, no fue posible la instalación de balón de contrapulsación aórtico; administrándose $4 \mathrm{ml}$ milrinona al 0,025\% nebulizada en 15 minutos, obteniendo mejoría de los parámetros hemodinámicos (Figura 1) permitiendo completar la disección de los planos quirúrgicos y canulación para la CEC. Se realizó puente safeno a LIMA y RVM sin incidentes.

\section{Discusión}

\section{Hipertensión pulmonar}

La HTP es definida como una elevación persistente de la PAP media (PAPM) en reposo de $25 \mathrm{mmHg}$ o más. En el 2018 fue categorizada por la Organización Mundial de la Salud (WHO) en 5 grupos determinados por su fisiopatología subyacente (Tabla 1)[1]. Desórdenes en la misma categoría WHO frecuentemente se presentan con cuadros clínicos y respuesta terapéutica similar[2].

\section{Fisiopatología de la hipertensión pulmonar}

La circulación pulmonar es un sistema de baja presión y baja resistencia con RVP normales que corresponden solo a un $10-20 \%$ de los valores de la circulación sistémica[2].

\section{Factores biológicos}

La HTP se asocia a un desbalance de diversas hormonas vasoactivas[2]. El metabolismo del ácido araquidónico en pacientes con HTP, se encuentra sesgado hacia la producción de tromboxano, un vasocons- trictor potente y agonista plaquetario. Por otro lado, la producción de óxido nítrico (NO), un vasodilatador producido a partir de la arginina por la enzima NO sintetasa se encuentra reducido.

La endotelina 1 (ET-1) es un 21 aminoácido peptídico producido en el músculo liso, células endoteliales y cardiomiocitos. Actúa como vasoconstrictor y estimula la proliferación de músculo liso vascular. Se han encontrado niveles aumentados de ET-1 en pacientes con HTP correlacionados con la severidad y pronóstico de la misma.

Por lo tanto, este desbalance de sustancias vasoactivas resulta en una vasoconstricción, proliferación celular y estado protrombótico crónico[2].

\section{Respuesta del VD a la sobrecarga de presión}

EI VD es una estructura de paredes delgadas, altamente complaciente, sensible a la postcarga y geométricamente adaptada para contraerse contra bajas resistencias de la circulación pulmonar.

La elevación crónica de las PAP estimula una hipertrofia concéntrica del VD como mecanismo adaptativo para la mantención del GC[1]. Esta hipertrofia hace al VD más susceptible a episodios isquémicos, al aumentar el consumo de oxígeno y hacerlo dependiente del flujo coronario diastólico determinado por el gradiente de presiones entre la arteria aorta y el VD[6].

La evolución natural de la enfermedad ante elevaciones crónicas de las PAP, no manejadas adecuadamente, es la dilatación del VD con insuficiencia tricúspidea y, finalmente, falla cardiaca derecha.

Este ciclo es perpetuado, además, por el shift del septum intraventricular hacia la izquierda, determinando un menor llene del VI, y por lo tanto, una disminución del GC con hipoperfusión tisular sistémica[1].

La elevación aguda de las PAP no permite la ins- 
Tabla 1. Clasificación de la Organización Mundial de la Salud de la hipertensión pulmonar (2008)[5]

\begin{tabular}{|c|c|c|c|}
\hline Grupo & Descripción & Ejemplos & Hemodinamia \\
\hline 1 & $\begin{array}{l}\text { Hipertensión arterial pul- } \\
\text { monar }\end{array}$ & $\begin{array}{l}\text { Idiopática, hereditaria, aso- } \\
\text { ciada a fármacos y tóxicos, } \\
\text { asociada con enfermedades } \\
\text { tejido conectivo, VIH, HTP } \\
\text { persistente del recién nacido }\end{array}$ & $\begin{array}{l}\text { PAPM }>25 \mathrm{mmHg} \\
\text { PCWP }<15 \mathrm{mmHg} \\
\mathrm{RVP}>240 \text { dinas } \bullet \bullet \mathrm{cm}^{-5}\end{array}$ \\
\hline 2 & $\begin{array}{l}\text { HTP debida a cardiopatía } \\
\text { izquierda }\end{array}$ & $\begin{array}{l}\text { Enfermedad valvular severa } \\
\text { mitral y/o aórtica, falla car- } \\
\text { diaca izquierda }\end{array}$ & $\begin{array}{l}\text { PAPM }>25 \mathrm{mmHg} \\
\text { PCWP }>15 \mathrm{mmHg} \\
\text { RVP }<240 \text { dinas } \bullet \bullet \mathrm{cm}^{-5}\end{array}$ \\
\hline 3 & $\begin{array}{l}\text { HTP debida a enfermedad } \\
\text { pulmonar y/o hipoxemia }\end{array}$ & $\begin{array}{l}\text { Enfermedad pulmonar obs- } \\
\text { tructiva crónica, enfermedad } \\
\text { pulmonar intersticial, hipo- } \\
\text { ventilación alveolar }\end{array}$ & $\begin{array}{l}\text { PAPM }>25 \mathrm{mmHg} \\
\text { PCWP }<15 \mathrm{mmHg} \\
\text { RVP }>240 \text { dinas } \bullet \cdot \mathrm{cm}^{-5}\end{array}$ \\
\hline 4 & $\begin{array}{l}\text { HTP tromboembólica crónica } \\
\text { y otras obstrucciones de las } \\
\text { arterias pulmonares }\end{array}$ & $\begin{array}{l}\text { Obstrucción trombótica de } \\
\text { las arterias pulmonares }\end{array}$ & $\begin{array}{l}\text { PAPM }>25 \mathrm{mmHg} \\
\text { PCWP }<15 \mathrm{mmHg} \\
\text { RVP }>240 \text { dinas } \bullet \cdot \mathrm{cm}^{-5}\end{array}$ \\
\hline 5 & $\begin{array}{l}\text { HTP de mecanismo no esta- } \\
\text { blecido y/o multifactorial }\end{array}$ & $\begin{array}{l}\text { Desórdenes mieloproliferati- } \\
\text { vos, sarcoidosis, neurofibro- } \\
\text { matosis, vasculitis, enferme- } \\
\text { dad tiroidea, enfermedad } \\
\text { renal crónica }\end{array}$ & $\begin{array}{l}\text { PAPM }>25 \mathrm{mmHg} \\
\text { PCWP }<15 \mathrm{mmHg} \\
\text { RVP }>240 \text { dinas } \bullet \cdot \mathrm{cm}^{-5}\end{array}$ \\
\hline
\end{tabular}

tauración de mecanismos compensatorios, por lo que generará una caída abrupta de la fracción de eyección del VD y dilatación VD, disminuyendo la precarga del ventrículo izquierdo y finalmente una disminución del GC sistémico[6],[7].

\section{Tratamiento}

El tratamiento de la HTP está determinado por la clasificación WHO. El manejo de aquellos pacientes dentro de la categoría 1 difiere significativamente del resto, por lo que lo analizaremos por separado.

\section{Hipertensión arterial pulmonar}

En las últimas décadas, se han descubierto múltiples terapias específicas para el manejo de la HTP arterial. Los antagonistas del receptor de endotelina como bosentan, ambrisentan y sitaxetan son recomendados en aquellos pacientes con CF II-III. Dentro de sus efectos adversos frecuentes se encuentra flushing, elevación de enzimas hepáticas y edema periférico.

Los inhibidores de la fosfodiesterasa 5 (sildenafil, tadalafil, vardenafil), aumentan la disponibilidad de NO al inhibir su metabolización y son de utilidad en pacientes con CF II-III. Prostacicilinas sintéticas como iloprost y treprostinil son reservadas para pacientes con CF III-IV[1].
Habitualmente, los pacientes con HTP arterial, reciben una terapia multimodal siendo la asociación de antagonistas receptor endotelina 1 e inhibidores de la fosfodiesterasa 5 las más utilizadas[2].

\section{HTP no hipertensión pulmonar arterial}

El manejo ambulatorio de las patologías no HTP arterial, es de características de soporte y adaptadas a la causa subyacente que gatilló la HTP. La HTP secundaria a patologías cardiacas izquierdas se manejan principalmente, con terapia médica intensiva basada en betabloqueadores, inhibidores de la angiotensina, antagonistas de la aldosterona, diuréticos de asa y vasodilatadores[2].

En cuanto al uso de los fármacos específicos para la HTP arterial en este grupo de pacientes, aún no hay evidencia clara de su efectividad.

La HTP es una complicación frecuente de la enfermedad valvular mitral y esta se ve, generalmente, exacerbada, secundaria a la injuria isquemia- reperfusión, inflamación y disfunción endotelial relacionada a la CEC[2]. La CEC prolongada, HTP preexistente, protección miocárdica subóptima y embolia coronaria, aumentan el riesgo de disfunción ventricular derecha (VD) durante la cirugía cardiaca. Se ha reportado hasta un $37-90 \%$ de mortalidad perioperatoria en pacientes que presentan falla VD[8].

El manejo perioperatorio tradicional de los pacien- 
tes con HTP incluye, el evitar factores gatillantes y la administración intravenosa de vasodilatadores como nitroglicerina, nitroprusiato de sodio, análogos de prostaciclinas y milrinona. Sin embargo, su uso se ve limitado por su efecto vasodilatador no selectivo causando hipotensión, disminución de presión de perfusión coronaria, isquemia miocárdica y finalmente falla cardiaca derecha[8],[9].

La administración de drogas por vía inhalatoria, parece ser ventajoso por las grandes cantidades de droga que se pueden administrar selectivamente a la circulación pulmonar. Estrategias clínicas por vía inhalatoria, incluyen el uso de óxido nítrico, milrinona y análogos de prostaciclina.

El óxido nítrico inhalado (ONi), ha sido usado ampliamente como vasodilatador pulmonar en cirugía cardiaca, pero debido a su toxicidad potencial, requiere de un equipo especializado para su administración, encareciendo y a su vez limitando su utilización[9]-[11]. Además, hay reportes que indican una inhibición de la agregación plaquetaria por el ONi que debe tenerse en cuenta, especialmente en el perioperatorio[12]. Los análogos de las prostaciclinas como el epoprostenol e iloprost se han relacionado igualmente con disfunción plaquetaria y efecto rebote tras la suspensión de su administración.

La milrinona es un inodilatador, que actúa incrementando los niveles de AMP cíclico en el músculo liso vascular y cardiaco secundario a la inhibición de la fosfodiesterasa III. El aumento del AMPc a su vez, produce un aumento del calcio intracelular resultando en un aumento de la contractilidad miocárdica y vasodilatación sistémica.

Su administración por vía inhalatoria ha demostrado actuar como un vasodilatador pulmonar selectivo. Wang et al.[4], compararon la administración de milrinona endovenosa versus vía inhalatorio en pacientes con HTP sometidos a RVM y encontraron que si bien en ambos grupos se producía disminución de PAPM y RVP, en el grupo de milrinona inhalada la presión arterial media (PAM) y resistencia vascular sistémica (RVS) eran significativamente más alta. Haralsson SA et al[9], encontraron que la milrinona inhalada disminuía la RVP hasta en un 20\%, sin afectar la RVS, a mismo Sablotzki et al, no encontrar disminución significativa de la RVSI.

El reciente estudio clínico randomizado de Taanver et al[8], comparó el efecto de la milrinona inhalada (50 mcg/kg), versus placebo, mostrando disminución de la PASP y PAPM sin efecto sobre la RVS. Gebhard et al, reportaron una disminución de PAP sistólica, reversión de la dilatación VD, disminución insuficiencia tricúspidea, aumento del GC y una disminución del requerimiento de drogas vasoactivas[13].

En este caso, se produjo una disminución de $13,25 \%$ de la PAPM a los 40 minutos tras la nebulización de $1 \mathrm{mg}$ milrinona en 15 minutos. Lo anterior concuerda con los hallazgos de Sablotzki $A$ et, al y Taanver et al, con una disminución de $13 \%$ y $16 \%$ de la PAP en pacientes con HTP respectivamente[8],[14]. En el estudio de Haraldsson et al, la disminución de PAPM, tras uso de $1 \mathrm{mg} / \mathrm{ml}$ a $0,3 \mathrm{ml} / \mathrm{min}$ por $10 \mathrm{mi}-$ nutos fue solo de un $6 \%$, sin embargo, su $n$ pequeño $(n=9)$ es un limitante de sus resultados.

En la paciente los efectos de vasodilatación pulmonar, comenzaron tras 20 minutos de administrada la nebulización, con un efecto máximo a los 40 minutos. La duración del efecto ha sido reportada por diferentes autores, Wang et al[4] mostró una vuelta a los valores basales de PAPM a los 60 min tras suspendida su administración de 4 horas duración $(1 \mathrm{mg} / \mathrm{ml}$ a 6 $\mathrm{ml} / \mathrm{h}$ ). Haraldsson et al, a su vez mostró una duración de 20 minutos tras una administración por 15 min[9]. Taanver et al[8] vio el retorno a los valores basales tras 0,5 horas.

El uso de milrinona inhalada, ha demostrado acortar la estadía en UCl, duración de la hospitalización y mortalidad intrahospitalaria en pacientes que presentaron falla VD intraoperatoria[13].

La milrinona inhalada se puede administrar a través de nebulizadores en jet, usando altos flujos de oxígeno (6-10 L/min) como en este caso, nebulizadores ultrasónicos, nebulización con malla de vibración e incluso como bolo intratraqueal[13],[15].

Si bien inicialmente se había descrito que los métodos de nebulización eran igualmente efectivos, recientemente se ha reportado una mayor entrega en la vía aérea distal del fármaco, con los nebulizadores en malla de vibración[16]. Es menos costosa y más fácil de administrar por inhalación que el NOi y los análogos de las prostaciclinas, y no tiene los efectos de rebote o hemorragia por disfunción plaquetaria.

Se ha descrito recientemente el uso de levosimendán, una molécula sensibilizadora de los canales de calcio con efecto inodilatador por vía inhalatoria[17]. Tannver et al, reportaron una disminución de la PAPS, PAPM y mantención de la RVS, que fueron comparables con la milrinona innalada. No obstante, la duración del efecto fue más prolongado tras la administración de levosimendán (3 horas vs 30 min en el grupo de milrinona inhalada)[17]. Hacen falta más estudios para esclarecer el rol de este fármaco prometedor. Sin embargo, por ahora su alto costo y baja disponibilidad limitan ampliamente su utilización. 


\section{Conclusiones}

El uso de milrinona inhalada, ha demostrado ser una herramienta útil para la disminución de la PAP, RVP, y mejoría del inotropismo, sin efectos sistémicos deletéreos. Su amplia disponibilidad, menor costo, facilidad de administración, incluso en pacientes no intubados, hacen pensar que podría ser una herra- mienta útil para el manejo en el perioperatorio de los pacientes con HTP. Sin embargo, hacen falta más trabajos para definir sus potencialidades.

\section{Conflictos de interés}

M. Escobar y F. Aranda declaran no tener conflictos de interés.

\section{Referencias}

1. Hosseinian L. Pulmonary hypertension and noncardiac surgery: implications for the anesthesiologist. J Cardiothorac Vasc Anesth. 2014 Aug;28(4):1064-74. https://doi. org/10.1053/j.jvca.2013.11.017 PMID:24675000

2. Thunberg CA, Gaitan BD, Grewal A, Ramakrishna H, Stansbury LG, Grigore AM. Pulmonary hypertension in patients undergoing cardiac surgery: pathophysiology, perioperative management, and outcomes. J Cardiothorac Vasc Anesth. 2013 Jun;27(3):551-72. https://doi. org/10.1053/j.jvca.2012.07.017 PMID:23036624

3. Lamarche $Y$, Perrault LP, Maltais $S$, Tétreault K, Lambert J, Denault AY. Preliminary experience with inhaled milrinone in cardiac surgery. Eur J Cardiothorac Surg. 2007 Jun;31(6):1081-7. https://doi. org/10.1016/j.ejcts.2007.02.019 PMID:17400468

4. Wang H, Gong M, Zhou B, Dai A. Comparison of inhaled and intravenous milrinone in patients with pulmonary hypertension undergoing mitral valve surgery. Adv Ther. 2009 Apr;26(4):462-8. https://doi.org/10.1007/s12325009-0019-4 PMID:19381522

5. Simonneau G, Gatzoulis MA, Adatia I, Celermajer D, Denton C, Ghofrani A, et al. [Updated clinical classification of pulmonary hypertension]. Turk Kardiyol Dern Ars. 2014 Oct;42 Suppl 1:45-54. PMID:25697033

6. Denault A, Deschamps A, Tardif JC, Lambert J, Perrault L. Pulmonary hypertension in cardiac surgery. Curr Cardiol Rev. 2010 Feb;6(1):1-14. https:// oi0.2174/157340310790231671 PMID:21286273

7. Strumpher J, Jacobsohn E. Pulmonary hypertension and right ventricular dysfunction: physiology and perioperative management. J Cardiothorac Vasc Anesth. 2011 Aug;25(4):687-704. https://doi. org/10.1053/j.jvca.2011.02.022 PMID:21620732

8. Kundra TS. The Effect of Inhaled Milrinone Versus Inhaled Levosimendan in Pulmonary Hypertension Patients Undergoing Mitral Valve Surgery- A PilotRandomized Double-Blind Study. In: V P, editor. Journal of Cardiothoracic and Vascular Anesthesia2018.

9. Haraldsson SA, Kieler-Jensen $\mathrm{N}$, Ricksten SE. The additive pulmonary vasodilatory effects of inhaled prostacyclin and inhaled milrinone in postcardiac surgical patients with pulmonary hypertension [table of contents.]. Anesth Analg. 2001 Dec;93(6):1439-45. https:// doi.org/10.1097/00000539200112000-00018 PMID:11726420

10. Fullerton DA, Mclntyre RC Jr.
Inhaled nitric oxide: therapeutic applications in cardiothoracic surgery. Ann Thorac Surg. 1996 Jun;61(6):1856-64. https://doi.org/10.1016/00034975(96)00046-X PMID:8651811

11. Blaise GA, Gauvin D, Gangal $M$, Authier S. Nitric oxide, cell signaling and cell death. Toxicology. 2005 Mar;208(2):177-92. https://doi. org/10.1016/j.tox.2004.11.032 PMID:15691583

12. Samama CM, Diaby M, Fellahi JL, Mdhafar A, Eyraud D, Arock M, et al. Inhibition of platelet aggregation by inhaled nitric oxide in patients with acute respiratory distress syndrome. Anesthesiology. 1995 Jul;83(1):56-65. https:// doi.org/10.1097/00000542199507000-00007 PMID:7605019

13. Gebhard CE, Rochon A, Cogan J, Ased H, Desjardins G, Deschamps A, et al. Acute Right Ventricular Failure in Cardiac Surgery During Cardiopulmonary Bypass Separation: A Retrospective Case Series of 12 Years' Experience With Intratracheal Milrinone Administration. J Cardiothorac Vasc Anesth. 2019 Mar;33(3):651-60. https://doi. org/10.1053/j.jvca.2018.09.016 PMID:30683595

14. Sablotzki A, Starzmann W, Scheubel R, Grond S, Czeslick EG. Selective pulmonary vasodilation with inhaled aerosolized 
milrinone in heart transplant candidates. Can J Anaesth. 2005 Dec;52(10):1076-82. https:// doi.org/10.1007/BF03021608 PMID:16326679

15. Gebhard CE, Desjardins G, Gebhard C, Gavra P, Denault AY. Intratracheal Milrinone Bolus Administration During Acute Right Ventricular Dysfunction After Cardiopulmonary Bypass. J Cardiothorac Vasc Anesth. 2017
Apr;31(2):489-96. https://doi. org/10.1053/j.jvca.2016.11.033 PMID:28216201

16. Gavra $P$, Laflamme $M$, Denault $A Y$, Théoret $Y$, Perrault LP, Varin $F$. Use of nebulized milrinone in cardiac surgery; Comparison of vibrating mesh and simple jet nebulizers. Pulm Pharmacol Ther. 2017 Oct;46:20-9. https://doi. org/10.1016/j.pupt.2017.08.004 PMID:28782710
17. Kundra TS, Prabhakar V, Kaur P, Manjunatha N, Gandham R. The Effect of Inhaled Milrinone Versus Inhaled Levosimendan in Pulmonary Hypertension Patients Undergoing Mitral Valve Surgery - A Pilot Randomized Double-Blind Study. J Cardiothorac Vasc Anesth. 2018 Oct;32(5):2123-9. https://doi. org/10.1053/j.jvca.2018.04.022 PMID:30098861 